\title{
Eating and electroencephalographic activity following orbital frontal stimulation in rats
}

\author{
JAN D. D. CIOE, BRYAN KOLB, and IAN Q. WHISHAW \\ University of Lethbridge, Lethbridge, Alberta TIK 3M4, Canada
}

\begin{abstract}
Periodic electrical stimulation of the sulcal frontal cortex produced a pronounced increase in food consumed in a 1-h session as compared with both a control nonstimulation period and stimulation of the medial frontal cortex, which did not elicit feeding. Stimulation of the sulcal cortex at slightly higher currents produced behavioral and electrographic seizure activity followed by eating during the postictal period. These results support the suggested role for the sulcal (orbital) frontal cortex in feeding and complement those obtained from a number of other lines of inquiry.
\end{abstract}

A number of studies have shown that orbital frontal cortex lesions disrupt feeding behavior (Brandes \& Johnson, 1978; Braun, 1975; Kolb \& Nonneman, 1975; Whishaw, Schallert, \& Kolb, in press). Although there are important differences between the effects of lesions to the orbital frontal cortex and the lateral hypothalamic nucleus on feeding and drinking (see Whishaw et al., in press, for details), there are sufficient similarities to suggest that each makes an important contribution to the organization of feeding behavior (Kolb, Nonneman, \& Whishaw, 1978; Kolb, Whishaw, \& Schallert, 1977; Whishaw, Schallert, \& Kolb, in press). Although it is well known that stimulation of the lateral hypothalamus induces eating and drinking in rats (e.g. Hoebel \& Teitelbaum, 1962; Margules \& Olds, 1962), there have been no reports of these behaviors with electrical stimulation of the orbital frontal cortex. If the orbital frontal cortex and the hypothalamus do indeed form an interrelated circuit involved in feeding, one might expect feeding to be elicited from stimulation of the orbital frontal cortex but not from stimulation of adjacent cortical regions (such as the medial frontal cortex), which have not been implicated in feeding behavior (Kolb, 1974). On the other hand, stimulation of the orbital frontal cortex has been shown to suppress locomotor activity in rats (Wilcott, 1979), so that one could also predict suppression of eating as part of a lowering of general activation following orbital frontal stimulation. Accordingly, this report describes the feeding behavior of rats with orbital frontal or medial frontal stimulation. In view of the report by Wishart, Bland, Vanderwolf, and Altman (1973) that stimulus-

This research was supported by NSERC grants to Bryan Kolb and Ian Q. Whishaw. We wish to thank Adria Allen for typing the manuscript. Jan D. D. Cioe is at the College of New Caledonia, 3330-22 Avenue, Prince George, British Columbia V2N 1P8, Canada. Reprint requests should be sent to Bryan Kolb, Department of Psychology, University of Lethbridge, Lethbridge, Alberta T1K 3M4, Canada. bound eating can result from stimulus-induced electrographic (EEG) seizures, EEG recordings were taken during stimulation tests in some of the rats in order to determine if the stimulation induced electrographic as well as behavioral seizures.

\section{METHOD}

\section{Subjects}

Subjects were six adult male hooded rats weighing approximately $350 \mathrm{~g}$ at the time of surgery. The animals were implanted either with stimulating electrodes bilaterally in the sulcal cortex $(n=3)$ or with one electrode in the medial cortex and one in the sulcal cortex on the contralateral side $(n=3)$. The latter three rats were also implanted with recording electrodes bilaterally in the hippocampus.

\section{Surgery}

The animals were anesthetized with sodium pentobarbital $(50 \mathrm{mg} / \mathrm{kg})$ and were given $1 \mathrm{mg} / \mathrm{kg}$ atropine sulphate prior to surgery.

The stimulating electrodes were bipolar twisted electrodes 140 microns in diameter, which were insulated except for the cross section at the tip. One electrode was cut $.5 \mathrm{~mm}$ shorter than the other. The electrodes were implanted in the orbital cortex using the following coordinates: $3.5 \mathrm{~mm}$ lateral to the midline, $3.0 \mathrm{~mm}$ anterior to bregma, and $6.2 \mathrm{~mm}$ ventral from the surface of the skull. The electrodes were implanted in the medial cortex using the following coordinates: $.75 \mathrm{~mm}$ lateral from the midline, $2.0 \mathrm{~mm}$ anterior to bregma, and $4.0 \mathrm{~mm}$ ventral from the surface of the skull. The incisor bar was adjusted to put lambda and bregma in the same horizontal plane.

The EEG recording electrodes were implanted at the same time as the stimulating electrodes. The electrode wires were 250 micron in diameter, insulated with Teflon except at the tip, and soldered to Winchester subminiature connectors. Two wires were implanted parallel to one another, with one tip cut $.5-1.0 \mathrm{~mm}$ shorter than the other in order to maximize the hippocampal record (Bland \& Whishaw, 1976). Electrodes were placed bilaterally in the hippocampus with the skull horizontal using coordinates of $4.0 \mathrm{~mm}$ posterior to bregma, $2.5 \mathrm{~mm}$ lateral to the midline, and $3.3 \mathrm{~mm}$ ventral from the skull. A Winchester subminiature component soldered to the head of a stainless steel screw was fixed to the interparietal bone to serve as a ground connection.

At the conclusion of the study, the animals. were deeply anesthetized with sodium pentobarbital and perfused intra- 
cardially with normal saline followed by $10 \%$ Formalin in saline. The brains were frozen at 40 microns and stained with cresyl violet.

\section{Procedure}

The rats were adapted to the wooden test box $(30 \times 30 \mathrm{x}$ $45 \mathrm{~cm}$ long with a Plexiglas front) for $1 \mathrm{~h} /$ day for 2 days prior to stimulation in order to habituate exploratory behavior. A high-fat diet consisting of a mixture of lard (30\% by weight), corn oil $(10 \%)$, sucrose $(33 \%)$, casein $(20 \%)$, plus vitamins and salt was provided in a 4-cm-high glass jar affixed to the test box. Water was provided in a Richter tube. The animals were fed the high-fat diet in the home cage for 1 week prior to testing in order to familiarize the animals with the food.

The stimulation testing was carried out using a $100-\mathrm{Hz}$ biphasic square wave (.1-msec pulse duration). The stimulating current was gradually increased from 50 microA, using a schedule of $30 \mathrm{sec}$ on, $30 \mathrm{sec}$ off, to a maximum of 400 microA or until the eating or seizure threshold was reached, whichever came first. This procedure was repeated on Day 2. The rats that showed elicited behavior were given four testing sessions in which they received 10 stimulations ( $30 \mathrm{sec}$ on, $30 \mathrm{sec}$ off) on Days 1 and 4 and 10 "control, 0 microA, stimulations" ( $30 \mathrm{sec}$ on, $30 \mathrm{sec}$ off) on Days 2 and 3. The amount of food consumed was recorded and the occurrence of eating, drinking, or related behaviors (chewing, jaw movements) was recorded for each 30-sec period.

Difference recordings of brain activity were made with a Grass ink-writing polygraph with half-amplitude points adjusted to 1 and $35 \mathrm{~Hz}$. The brain electrodes were connected to preamplifiers by a light flexible cable. Records were taken during the entire stimulation session, and notes were written on the polygraph paper to enable later correlation of the EEG with behavior.

\section{RESULTS}

\section{Histology}

The stimulating electrodes aimed at the orbital cortex were located in the orbital cortex and underlying white matter, with the exception of one ineffective electrode that apparently left the brain and was lodged against the skull. The medial electrodes were all in the medial frontal cortex (see Figure 1).

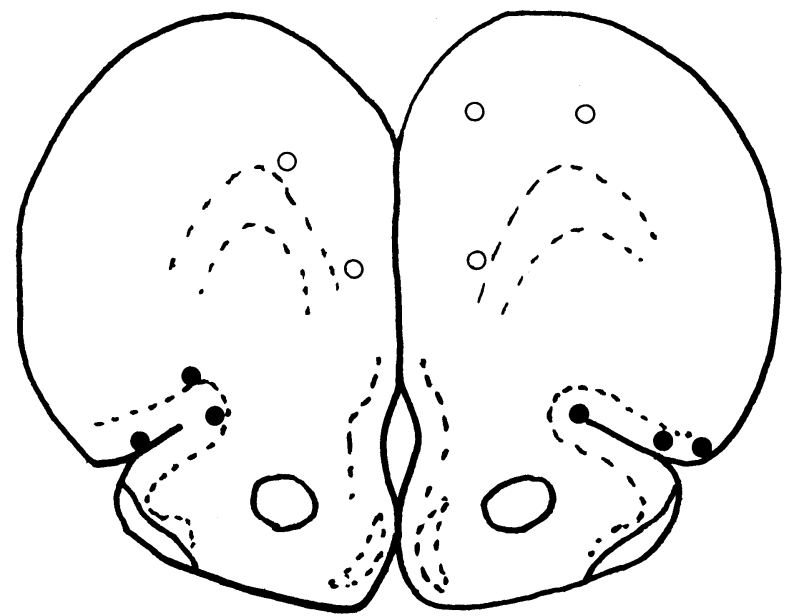

Figure 1. Composite figure showing location of the stimulating electrodes in three rats with both stimulating and recording electrodes.
Table 1

Summary of Stimulation Results

\begin{tabular}{lcccc}
\hline & \multicolumn{3}{c}{ Stimulus } \\
\cline { 2 - 4 } & On & Off & Total & AC \\
\hline Stimulation Days & $10.8^{*}$ & $21.0^{*} \dagger$ & $31.8^{*}$ & $18.83^{*}$ \\
Control Days & 4.8 & 4.0 & 8.8 & 3.79 \\
\hline
\end{tabular}

Note-Numbers in the "Stimulus On" and "Stimulus Off" columns represent total number of 30-sec periods, during which eating was observed at least once (maximum $=30$ ). AC=amount consumed (in grams). $\quad$ *Stimulation days differ significantly $(p<.01)$ from control days. TStimulus "off" differs significantly $(p<.05)$ from stimulus "on" on stimulation days.

\section{Behavior and EEG}

Stimulation of the medial frontal cortex was without discernible effect on elicited behavior, whereas stimulation of the orbital cortex produced eating from eight of the nine electrodes. It can be seen in Table 1 that there was significantly more eating observed on stimulation days than on control days (Mann-Whitney Us $=0$, $\mathrm{p}<.01$ ), both in terms of amount of food eaten and the number of stimulation periods producing eating. Rather than being bound only to the electrical stimulation, eating also occurred during interstimulation intervals; indeed, there was significantly more eating in the periods following the stimulation than during it (Mann-Whitney Us $=3, p<.05)$. The current thresholds for elicited eating were higher (mean $=140$ microA, range $=60$ 220 microA) than those usually reported for stimulation in the hypothalamus (Cioe, 1978).

In addition to eliciting eating, the stimulation produced motor seizures in all six animals, and clear electrographic seizures were recorded in the three rats with hippocampal recording electrodes. The motor seizures ranged from a sudden behavioral arrest to various degrees of head displacement, loss of balance, and gross movements involving tonic-clonic jerks of the limbs, head, and body. The EEG recordings showed that the electrographic seizures were usually bilateral, but sometimes electrographic seizuring occurred only on the side ipsilateral to the stimulation, and often slowly spread to the contralateral hippocampus (see Figure 2). Electrographic seizure activity frequently continued after stimulus offset, but at currents that appear near the seizure threshold, seizure activity terminated when the stimulation ended.

Although both the eating and electrographic and behavioral seizures occurred on stimulation days, the electrographic and behavioral seizures seldom occurred until the end of the sessions, whereas eating usually began with the first or second stimulation. Further, the threshold for electrographic or behavioral seizuring was sometimes three or four times higher than the threshold for eating (e.g., 80 vs. 250 microA, 100 vs. 250 microA, 50 vs. 100 micro $A$, for the three rats with recording electrodes). 
A

L hpc

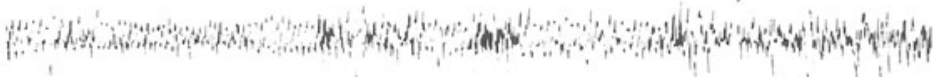

R hpc

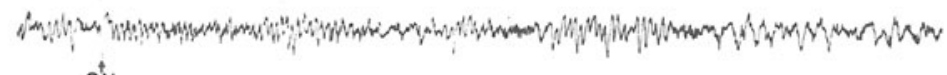
ON

L hpc

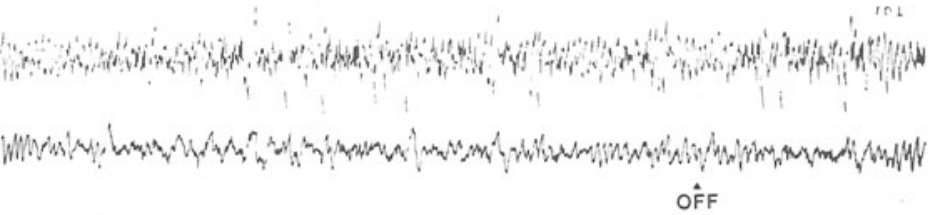

R hpc

$$
\text { OFF }
$$

L hpc

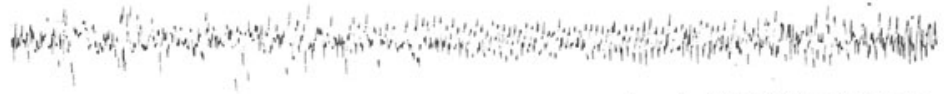

R hpc

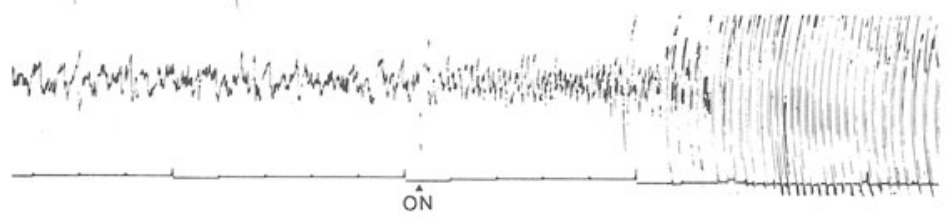

L hpc

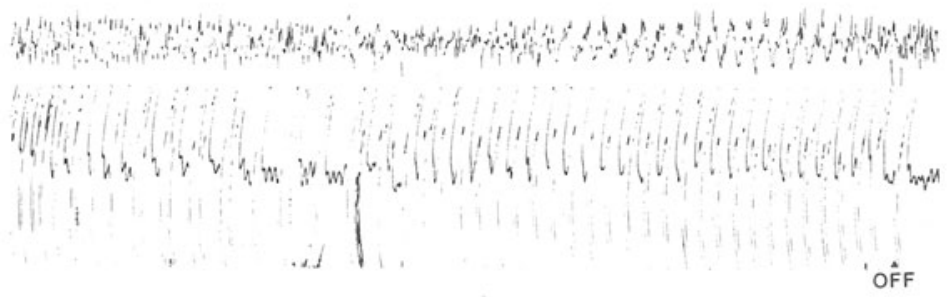

L hpc

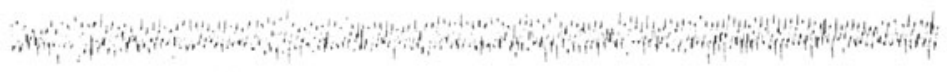

R hpc

Figure 2. (A) Hippocampal EEG record of a rat during 50-microA stimulation of the orbital frontal cortex. This stimulation produced eating during and after the stimulation. The bottom two tracings continue from the top tracings. Stimulation began at $\mathrm{ON}$ and terminated at OFF. (B) Hippocampal EEG record of the same rat during 100-microA stimulation of the same electrode. Note the seizure activity that began in the right hippocampus and spread to the left hippocampus. Seizure activity terminated when the stimulation was turned off. The rat had rhythmical movements of the head and forepaws during the electrographic seizure and ate during the postictal period (bottom tracing). Calibration: 1 sec; 500 microV.

\section{DISCUSSION}

The results of this experiment support the hypothesis that the orbital frontal cortex plays a role in the organization and control of feeding behavior. Periodic electrical stimulation of this area produced a pronounced increase in food consumed in a 1-h test session as compared with a 1-h control nonstimulation period. The amount of food consumed during the stimulation session approximated the rat's daily intake for a 24 -h period. Electrical stimulation of the adjacent medial frontal cortex produced no increase in feeding. These results support a role for the orbital frontal cortex in feeding and complement those obtained from a number of other lines of inquiry. First, lesions of orbital frontal cortex produce acute aphagia and adipsia and chronic weight loss accompanied or resulting from disabilities in oral manipulatory movement used for eating and drinking (Kolb \& Nonneman, 1975). Second, self-stimulation has been obtained from the orbital frontal cortex (Mora, 1978; Rolls \& Cooper, 1974) as well as from other brain areas that produce electrically elicited eating (Margules \& Olds, 1962). Third, many types of brain damage producing aphagia and adipsia typically also destroy cells, fibers, or terminals of dopamine systems (Marshall, Richardson, \& Teitelbaum, 1974; Ungerstedt, 1971). Since there are dopamine terminals within orbital frontal cortex (Fuxe, Hokfelt, Johansson, Jonsson, Lidbrink, \& Ljungdahl, 1974), it seems possible that stimulation of them can produce feeding such as that observed in this study, or aphagia consequent to lesions. However, the demonstration that orbital frontal cortex stimulation produces eating also raises three rather specific questions: (1) Is the eating similar to that which follows 
lateral hypothalamic stimulation? (2) What neural changes underlie the elicitation of eating? (3) Is the feeding analogous to "normal" feeding?

Feeding elicited by lateral hypothalamic stimulation is usually stimulus bound: Eating begins with the onset of stimulation and terminates when or shortly after stimulation ends (Hess, 1954; Hoebel \& Teitelbaum, 1962; Margules \& Olds, 1962). In response to orbital frontal stimulation the rats ate during both the 30 -sec stimulation and the following 30-sec nonstimulation periods. In fact, more eating occurred during interstimulus intervals. Thus, orbital frontal cortex elicited eating is different from lateral hypothalamic elicited eating at least in this important way. It may be that orbital cortex stimulation produces longer lasting effects because it initiates or triggers feeding bouts in a manner analogous to those that occur in response to a period of food deprivation. Such an interpretation is consistent with a traditional view that the cortex can initiate behaviors on motor programs that outlast the eliciting stimulus (e.g., Vanderwolf, Kolb, \& Cooley, 1978). It is possible, however, that poststimulation feeding has other causes, such as abnormalities or changes of activity in other brain areas.

To assess brain normalcy during stimulation, hippocampal EEG was recorded, since it has proved a sensitive index to the effects of brain stimulation (Whishaw, Bland, \& Vanderwolf, 1972; Whishaw \& Nikkel, 1975). Orbital frontal stimulation produced no consistent alterations in EEG in the hippocampus, but after a number of stimulations, particularly at higher current intensities, epileptiform or electrographic seizures occurred in the hippocampus. Thus in addition to its more direct effects, stimulation may have produced eating indirectly through abnormal activation of other brain structures. Both septal stimulation (Wishart et al., 1973) and hippocampal stimulation (Milgram, 1969) have also been reported to produce eating that is secondary to elicited electrographic discharges. At present it is unknown how epileptiform activity causes eating, but it may be via release of transmitter or hormonal substances in sites such as the hypothalamus to which hippocampal efferents project.

To date we have not attempted to determine whether orbital frontal stimulation elicited eating is similar to spontaneous eating. Schallert (1977) has reported that lateral hypothalamic stimulation does not cause rats to investigate a source of food odors in the manner of food-deprived rats. This suggests that lateral hypothalamic stimulation does not make rats hungry or produce eating that is driven by hunger. Similar experiments might be used effectively with orbital frontal stimulation to further clarify the nature of this cortically elicited eating.

Additionally, it would also be worthwhile determining whether this cortically elicited eating can occur in the absence of stimulation-induced electrographic seizures. Possibly, antiepileptic drugs such as phenobarbital would block electrographic discharges but not feeding. However, in the absence of such studies, the present results considered together with previous lesion work and pharmacological work give further support to the idea that the orbital frontal cortex is involved in the feeding activities of the rat.

\section{REFERENCES}

Bland, B. H., \& Whishaw, I. Q. Generators and topography of hippocampal theta (RSA) in anesthetized and freely moving rats. Brain Research, 1976, 118, 259-280.

Brandes, J. S., \& Johnson, A. K. Recovery of feeding in rats following frontal neocortical ablations. Physiology \& Behavior, 1978, 20, 763-770.

Braun, J. J. Neocortex and feeding in the rat. Journal of Comparative and Physiological Psychology, 1975, 89, 507-522.

CIOE, J. D. D. Electrically elicited feeding: Dissociation from "natural" feeding and catecholamine involvement (Doctoral dissertation, University of Western Ontario, 1978). Dissertation Abstracts International, 1979, 39, 4608B-4609B.
Fuxe, K., Hokfelt, T., Johansson, O., Jonsson, G., Lidbrink, P., \& Ljungdahl, A. The origin of dopamine nerve terminals in the limbic and frontal cortex. Evidence for meso-cortico dopamine neurons. Brain Research, 1974, 82, 349-355.

Hess, W. R. Diencephalon: Autonomic and extra-pyramidal functions. New York: Grune \& Stratton, 1954.

Hoebel, B. G., \& Teitelbaum, P. Hypothalamic control of feeding and self-stimulation. Science, 1962, 135, 375-377.

KoLB, B. Prefrontal lesions alter eating and hoarding behavior in rats. Physiology \& Behavior, 1974, 12, 507-511.

Kolb, B., \& Nonneman, A. J. Prefrontal cortex and the regulation of food intake in the rat. Journal of Comparative and Physiological Psychology, 1975, 88, 806-815.

Kolb, B., Nonneman, A. J., \& Whishaw, I. Q. Influence of frontal neocortex lesions and body weight manipulation on the severity of lateral hypothalamic aphagia. Physiology \& Behavior, 1978, 21, 541-547.

Kolb, B., Whishaw, I. Q., \& Schalle RT, T. Aphagia, behavior sequencing and body weight set point following orbital frontal lesions in rats. Physiology \& Behavior, 1977, 19, 93-103.

Lindvall, O., Buorklund, A., Moore, R. Y., \& Stenevi, U. Mesencephalic dopamine neurons projecting to neocortex. Brain Research, 1974, 81, 325-331.

Margules, D. L., \& OLDS, J. Identical “feeding" and "reward" systems in the lateral hypothalamus of rats. Science, 1962, 135, 374-375.

Marshall, J. F., Richardson, J. S., \& Teitelbaum, P. Nigro-striatal damage and the lateral hypothalamic syndrome. Journal of Comparative and Physiological Psychology, 1974, 87, 808-830.

Milgram, W. H. Effect of hippocampal stimulation on feeding in the rat. Physiology \& Behavior, 1969, 4, 665-670.

Mora, F., Avrith, D. B., \& Rolls, E. T. An electrophysiological and behavioural study of self-stimulation in the orbitoLife Science, 1978, 22, 919-930.

Mora, F., Avrith, D. B., \& Rolls, E. T. An electrophysiolological and behavioural study of self-stimulation in the orbitofrontal cortex of the rhesus monkey. Brain Research Bulletin, $1980,5,111-115$.

Rolls, E. T., \& Cooper, S. J. Anesthetization and stimulation of the sulcal prefrontal cortex and brain-stimulation reward. Physiology \& Behavior, 1974, 12, 563-571.

Schalle RT, T. Reactivity to food odors during hypothalamic stimulation in rats not experienced with stimulation-induced eating. Physiology \& Behavior, 1977, 20, 1061-1066.

UNGERSTEDT, U. Adipsia and aphagia after 6-hydroxydopamine induced degeneration of the nigro-striatal dopamine system in the rat brain. Acta Physiologica Scandinavica (Supplement 367), 1971, 82, 95-122.

Vanderwolf, C. H., Kolb, B., \& Cooley, R. C. The behavior of the rat following removal of the neocortex and hippocampal formation. Journal of Comparative and Physiological Psychology, 1978, 92, 156-175.

Whishaw, I. Q., Bland, B. H., \& Vande Rwolf, C. H. Hippocampal activity, behavior, self-stimulation, and heart rate during electrical stimulation of the lateral hypothalamus. Journal of Comparative and Physiological Psychology, 1972, 79, 115-127.

Whishaw, I. W., \& Nikkel, R. W. Anterior hypothalamic electrical stimulation and hippocampal EEG in the rat: Suppressed EEG, locomotion, self-stimulation and inhibition of shock avoidance. Behavioral Biology, 1975, 13, 1-20.

Whishaw, I. Q., Schalle RT, T., \& Kolb, B. An analysis of feeding, sensory, and motor abilities of rats after decortication. Journal of Comparative and Physiological Psychology, in press.

WilcotT, R. C. Cortical stimulation and suppression of activity, in the rat. Physiology \& Behavior, 1979, 23, 1041-1048.

Wishart, T. B., Bland, B. H., Vanderwolf, C. H., \& Altman, J. L. Electroencephalographic correlates of behaviors elicited by electrical stimulation of the septum: Seizure induced feeding. Behavioral Biology, 1973, 9, 763-769.

(Received for publication September 16, 1980.) 